\title{
ON CLOSED MINIMAL SUBMANIFOLDS IN PINCHED RIEMANNIAN MANIFOLDS
}

\author{
HONG-WEI XU
}

\begin{abstract}
In this paper, we first prove a generalized Simons integral inequality for closed minimal submanifolds in a Riemannian manifold. Second, we prove a pinching theorem for closed minimal submanifolds in a complete simply connected pinched Riemannian manifold, which generalizes the results obtained by S. S. Chern, M. do Carmo, and S. Kobayashi and A. M. Li and J. M. Li respectively. Finally, we obtain a distribution theorem for the square norm of the second fundamental form of $M$ under the assumption that $M$ is a minimal submanifold with parallel second fundamental form in a Riemannian manifold.
\end{abstract}

\section{INTRODUCTION}

Let $M^{n}$ be an $n$-dimensional oriented closed minimal submanifold in an $(n+p)$-dimensional manifold $N^{n+p}$. We denote the square norm of the second fundamental form of $M$ by $S$. In the case that the ambient manifold $N$ is the Euclidean sphere $S^{n+p}(1)$, it is well known [2] that if $S \leq n /(2-1 / p)$ on $M$, then either $M$ is the unit sphere $S^{n}(1)$, one of the Clifford minimal hypersurfaces in $S^{n+1}(1)$, or the Veronese surface in $S^{4}(1)$. Further discussions in this regard have been carried out by many other authors $([3,5,8,9,12]$, etc.). Recently, A. M. Li and J. M. Li [6] have improved the pinching constant above to $\frac{2}{3} n$ for the case $p \geq 3$. But all these results were obtained under the assumption that the ambient manifolds possess very nice symmetry.

The aim of the present paper is to establish a generalized Simons integral inequality for minimal submanifolds in a Riemannian manifold, and prove a pinching theorem for minimal submanifolds in a complete simply connected pinched Riemannian manifold, which does not possess symmetry in general. The proof uses some equations and inequalities naturally associated to the second fundamental form of $M$, the curvature tensor of $N$, and their covariant derivatives. Since we do not assume that $N^{n+p}$ is a sphere, the maximum principle and the estimate for $\Delta S$ in $[2,6]$ cannot be applied here, and the trick of constructing a differentiable 1 -form and using integral estimates seems essential. Finally, a distribution theorem for $S$ is obtained under the assumption

Received by the editors June 21, 1993 and, in revised form, October 28, 1993; originally communicated to the Proceedings of the AMS by Peter Li.

1991 Mathematics Subject Classification. Primary 53C40; Secondary 53C20.

Key words and phrases. Closed minimal submanifold, pinched Riemannian manifold.

Research partially supported by the National Natural Science Foundation of China, and the Natural Science Foundation of Zhejiang Province. 
that $M$ is a minimal submanifold with parallel second fundamental form in a Riemannian manifold.

\section{Preliminaries}

Let $M^{n}$ be an $n$-dimensional Riemannian manifold immersed in an $(n+p)$ dimensional Riemannian manifold $N^{n+p}$. We shall make use of the following convention on the range of indices:

$$
\begin{gathered}
1 \leq A, B, C, \ldots \leq n+p, \quad 1 \leq i, j, k, \ldots \leq n, \\
n+1 \leq \alpha, \beta, \gamma, \ldots \leq n+p .
\end{gathered}
$$

Choose a local field of orthonormal frames $\left\{e_{A}\right\}$ in $N$ such that, restricted to $M$, the $e_{i}$ 's are tangent to $M$. Let $\left\{\omega_{A}\right\}$ and $\left\{\omega_{A B}\right\}$ be the field of dual frames and the connection 1-forms of $N$ respectively. Restricting these forms to $M$, we have

$$
\begin{gathered}
\omega_{\alpha i}=\sum_{j} h_{i j}^{\alpha} \omega_{j}, \quad h_{i j}^{\alpha}=h_{j i}^{\alpha}, \\
h=\sum_{\alpha, i, j} h_{i j}^{\alpha} \omega_{i} \otimes \omega_{j} \otimes e_{\alpha}, \quad \xi=\frac{1}{n} \sum_{\alpha, i} h_{i i}^{\alpha} e_{\alpha}, \\
R_{i j k l}=K_{i j k l}+\sum_{\alpha}\left(h_{i k}^{\alpha} h_{j l}^{\alpha}-h_{i l}^{\alpha} h_{j k}^{\alpha}\right), \\
R_{\alpha \beta k l}=K_{\alpha \beta k l}+\sum_{i}\left(h_{i k}^{\alpha} h_{i l}^{\beta}-h_{i l}^{\alpha} h_{i k}^{\beta}\right),
\end{gathered}
$$

where $h, \xi, R_{\alpha \beta k l}, R_{i j k l}$, and $K_{A B C D}$ are the second fundamental form, the mean curvature vector, the normal curvature tensor, the curvature tensor of $M$, and the curvature tensor of $N$ respectively. We define

$$
S=\|h\|^{2}, \quad H=\|\xi\|, \quad H_{\alpha}=\left(h_{i j}^{\alpha}\right)_{n \times n} .
$$

$M$ is called minimal if $H$ vanishes identically. Therefore, if $M$ is minimal, its scalar curvature is given by

$$
R=\sum_{i, j} K_{i j i j}-S .
$$

Now we define the covariant derivatives of $h_{i j}^{\alpha}$, denoted by $h_{i j k}^{\alpha}$ and $h_{i j k l}^{\alpha}$ respectively, as

$$
\begin{gathered}
\sum_{k} h_{i j k}^{\alpha} \omega_{k}=d h_{i j}^{\alpha}+\sum_{s} h_{s j}^{\alpha} \omega_{i s}+\sum_{s} h_{i s}^{\alpha} \omega_{j s}+\sum_{\beta} h_{i j}^{\beta} \omega_{\alpha \beta}, \\
\sum_{l} h_{i j k l}^{\alpha} \omega_{l}=d h_{i j k}^{\alpha}+\sum_{s} h_{s j k}^{\alpha} \omega_{i s}+\sum_{s} h_{i s k}^{\alpha} \omega_{j s}+\sum_{s} h_{i j s}^{\alpha} \omega_{k s}+\sum_{\beta} h_{i j k}^{\beta} \omega_{\alpha \beta} .
\end{gathered}
$$

Then we have

$$
h_{i j k}^{\alpha}-h_{i k j}^{\alpha}=K_{\alpha i k j}
$$

and the Ricci formula

$$
h_{i j k l}^{\alpha}-h_{i j l k}^{\alpha}=\sum_{s} h_{s j}^{\alpha} R_{s i k l}+\sum_{s} h_{i s}^{\alpha} R_{s j k l}+\sum_{\beta} h_{i j}^{\beta} R_{\beta \alpha k l} .
$$


Considering $K_{\alpha i j k}$ as a section of $T^{\perp}(M) \otimes T^{*}(M) \otimes T^{*}(M) \otimes T^{*}(M)$, we also define its covariant derivative $K_{\alpha i j k l}$ as

$$
\begin{aligned}
\sum_{l} K_{\alpha i j k l} \omega_{l}= & d K_{\alpha i j k}+\sum_{s} K_{\alpha s j k} \omega_{i s}+\sum_{s} K_{\alpha i s k} \omega_{j s} \\
& +\sum_{s} K_{\alpha i j s} \omega_{k s}+\sum_{\beta} K_{\beta i j k} \omega_{\alpha \beta} .
\end{aligned}
$$

$M$ is called a submanifold with parallel second fundamental form if $h_{i j k}^{\alpha} \equiv 0$ for all $i, j, k, \alpha$. The Laplacian $\Delta h_{i j}^{\alpha}$ of the second fundamental form $h$ is defined by $\Delta h_{i j}^{\alpha}=\sum_{k} h_{i j k k}^{\alpha}$. In the next section, we sometimes also use $\nabla_{k} h_{i j}^{\alpha}$ to denote $h_{i j k}^{\alpha}$, etc.

For a matrix $A=\left(a_{i j}\right)_{n \times n}$ we denote by $N(A)$ the square norm of $A$, i.e., $N(A)=\operatorname{tr}\left(A^{t} A\right)=\sum_{i, j} a_{i j}^{2}$. Then $N(A)=N\left(T A^{t} T\right)$, for each orthogonal $(n \times n)$-matrix $T$.

Proposition 1 (see $[2,6])$. Let $A_{n+1}, A_{n+2}, \ldots, A_{n+p}$ be symmetric $(n \times n)$ matrices. Denote $S_{\alpha \beta}=\operatorname{tr}\left(A_{\alpha}^{t} A_{\beta}\right), S_{\alpha}=S_{\alpha \alpha}=N\left(A_{\alpha}\right), S=\sum_{\alpha} S_{\alpha}$. Then

$$
\sum_{\alpha, \beta} N\left(A_{\alpha} A_{\beta}-A_{\beta} A_{\alpha}\right)+\sum_{\alpha, \beta} S_{\alpha \beta}^{2} \leq\left(1+\frac{1}{2} \operatorname{sgn}(p-1)\right) S^{2},
$$

where $\operatorname{sgn}(\cdot)$ is the standard sign function, and the equality holds if and only if at most two matrices $A_{\alpha}$ and $A_{\beta}$ are not zero and these two matrices can be transformed simultaneously by an orthogonal matrix into scalar multiples of $\tilde{A_{\alpha}}$ and $\tilde{A}_{\beta}$ respectively, where

$$
\widetilde{A}_{\alpha}=\left(\begin{array}{cc|c}
1 & 0 & 0 \\
0 & -1 & \\
\hline 0 & 0
\end{array}\right), \quad \tilde{A}_{\beta}=\left(\begin{array}{cc|c}
0 & 1 & 0 \\
1 & 0 & 0 \\
\hline 0 & 0
\end{array}\right) .
$$

Proposition 2 (see [4]). Let $N$ be an $(n+p)$-dimensional Riemannian manifold. If $a \leq K_{N} \leq b$ at $a$ point $x \in N$, then, at this point,

(i) $\left|K_{A C B C}\right| \leq \frac{1}{2}(b-a)$, for $A \neq B$.

(ii) $\left|K_{A B C D}\right| \leq \frac{2}{3}(b-a)$, for $A, B, C, D$ distinct with each other.

\section{INEQUALITIES AND PINCHING THEOREMS}

From now on, we assume that $M^{n}$ is a minimal submanifold in $N^{n+p}$. By (2.5), (2.6), and the minimality of $M$, we have

$$
\begin{aligned}
\Delta h_{i j}^{\alpha}= & -\sum_{k}\left(K_{\alpha k i k j}+K_{\alpha i j k k}\right)+\sum_{k, m} h_{m i}^{\alpha} R_{m k j k} \\
& +\sum_{k, m} h_{k m}^{\alpha} R_{m i j k}+\sum_{k, \beta} h_{k i}^{\beta} R_{\alpha \beta k j} .
\end{aligned}
$$

Substituting (2.3) and (2.4) into the above, (3.1) becomes

$$
\begin{aligned}
\Delta h_{i j}^{\alpha} & =-\sum_{k}\left(K_{\alpha k i k j}+K_{\alpha i j k k}\right)+\sum_{m, k}\left(h_{m i}^{\alpha} K_{m k j k}+h_{m k}^{\alpha} K_{m i j k}\right)+\sum_{k, \beta} h_{k i}^{\beta} K_{\alpha \beta k j} \\
& +\sum_{m, k, \beta}\left(h_{m i}^{\alpha} h_{m j}^{\beta} h_{k k}^{\beta}+2 h_{k m}^{\alpha} h_{k i}^{\beta} h_{m j}^{\beta}-h_{k m}^{\alpha} h_{k m}^{\beta} h_{i j}^{\beta}-h_{m i}^{\alpha} h_{m k}^{\beta} h_{k j}^{\beta}-h_{m j}^{\alpha} h_{k i}^{\beta} h_{m k}^{\beta}\right) .
\end{aligned}
$$


Therefore,

$$
\begin{aligned}
\frac{1}{2} \Delta S= & \sum_{i, j, k, \alpha}\left(h_{i j k}^{\alpha}\right)^{2}+\sum_{i, j, \alpha} h_{i j}^{\alpha} \Delta h_{i j}^{\alpha} \\
= & \sum_{i, j, k, \alpha}\left(h_{i j k}^{\alpha}\right)^{2}-\sum_{i, j, k, \alpha}\left(h_{i j}^{\alpha} K_{\alpha k i k j}+h_{i j}^{\alpha} K_{\alpha i j k k}\right) \\
& +\sum_{i, j, k, m, \alpha}\left(h_{m j}^{\alpha} h_{i j}^{\alpha} K_{m k i k}+h_{m k}^{\alpha} h_{i j}^{\alpha} K_{m i j k}\right) \\
& +\sum_{i, j, k, \alpha, \beta} h_{i j}^{\alpha} h_{k i}^{\beta} K_{\alpha \beta k j}-\sum_{i, j, k, l, \alpha, \beta} h_{i j}^{\alpha} h_{k l}^{\alpha} h_{i j}^{\beta} h_{k l}^{\beta} \\
& -\sum_{i, j, k, l, \alpha, \beta}\left(h_{i k}^{\alpha} h_{j k}^{\beta}-h_{j k}^{\alpha} h_{i k}^{\beta}\right)\left(h_{i l}^{\alpha} h_{j l}^{\beta}-h_{j l}^{\alpha} h_{i l}^{\beta}\right) .
\end{aligned}
$$

Put

$$
S_{\alpha \beta}=\sum_{i, j} h_{i j}^{\alpha} h_{i j}^{\beta}
$$

Then the $(p \times p)$-matrix $\left(S_{\alpha \beta}\right)$ is symmetric and can be assumed to be diagonal for a suitable choice of $\left\{e_{\alpha}\right\}$, i.e.,

$$
S_{\alpha \beta}=S_{\alpha} \delta_{\alpha \beta} \text { for all } \alpha, \beta \text {. }
$$

By the definition, $S=\sum_{\alpha} S_{\alpha}$. From (3.2) we have

\section{Lemma 1. Denote}

$$
\begin{aligned}
A & =-\sum_{\alpha, \beta} N\left(H_{\alpha} H_{\beta}-H_{\beta} H_{\alpha}\right)-\sum_{\alpha} S_{\alpha}^{2} \\
B & =\sum_{i, j, k, m, \alpha}\left(h_{m j}^{\alpha} h_{i j}^{\alpha} K_{m k i k}+h_{m k}^{\alpha} h_{i j}^{\alpha} K_{m i j k}\right)+\sum_{i, j, k, \alpha, \beta} h_{i j}^{\alpha} h_{k i}^{\beta} K_{\alpha \beta k j}, \\
C & =\sum_{i, j, k, \alpha}\left(h_{i j k}^{\alpha}\right)^{2}-\sum_{i, j, k, \alpha}\left(h_{i j}^{\alpha} K_{\alpha k i k j}+h_{i j}^{\alpha} K_{\alpha i j k k}\right) .
\end{aligned}
$$

Then

$$
\frac{1}{2} \Delta S=A+B+C .
$$

Let $a(x)$ and $b(x)$ denote the infimum and the supremum of the sectional curvature of $N$ at a point $x$ respectively. Now we derive a lower bound for $B$ in terms of $a, b$, and $S$.

Lemma 2. $B \geq n b S-\left[n+\frac{2}{3}(p-1)(n-1)^{1 / 2}\right](b-a) S$.

Proof. Fix a vector $e_{\alpha}$. Let $\left\{e_{i}\right\}$ be a frame diagonalizing the matrix $\left(h_{i j}^{\alpha}\right)$ such that

$$
h_{i j}^{\alpha}=\lambda_{i}^{\alpha} \delta_{i j}, \quad 1 \leq i, j \leq n .
$$

Then

$$
\begin{gathered}
\sum_{i, j, k, m} h_{m j}^{\alpha} h_{i j}^{\alpha} K_{m k i k}+\sum_{i, j, k, m} h_{m k}^{\alpha} h_{i j}^{\alpha} K_{m i j k}+\sum_{i, j, k, \beta} h_{i j}^{\alpha} h_{k i}^{\beta} K_{\alpha \beta k j} \\
=\sum_{i, k}\left(\lambda_{i}^{\alpha}\right)^{2} K_{i k i k}+\sum_{i, k} \lambda_{k}^{\alpha} \lambda_{i}^{\alpha} K_{k i i k}+\sum_{i, k, \beta} h_{k i}^{\beta} \lambda_{i}^{\alpha} K_{\alpha \beta k i}
\end{gathered}
$$


By Proposition 2, we have

$$
\left|K_{\alpha \beta k i}\right| \leq \frac{2}{3}(b-a) \text { for } \alpha \neq \beta, i \neq k .
$$

Hence, for fixed $\alpha$, one sees

$$
\begin{aligned}
\sum_{i, k, \beta} h_{k i}^{\alpha} \lambda_{i}^{\alpha} K_{\alpha \beta k i} \geq-\sum_{i \neq k, \beta \neq \alpha} \frac{2}{3}(b-a)\left|h_{k i}^{\beta} \lambda_{i}^{\alpha}\right| \\
\geq-\sum_{i \neq k, \beta \neq \alpha} \frac{1}{3}(b-a)\left[(n-1)^{1 / 2}\left(h_{k i}^{\beta}\right)^{2}+(n-1)^{-1 / 2}\left(\lambda_{i}^{\alpha}\right)^{2}\right] \\
\geq-\frac{1}{3}(n-1)^{1 / 2}(b-a) \sum_{\beta \neq \alpha} \operatorname{tr} H_{\beta}^{2} \\
\quad-\frac{1}{3}(n-1)^{1 / 2}(p-1)(b-a) \operatorname{tr} H_{\alpha}^{2} .
\end{aligned}
$$

On the other hand, we have

$$
\begin{aligned}
& \sum_{i, k}\left(\lambda_{i}^{\alpha}\right)^{2} K_{i k i k}+\sum_{i, k} \lambda_{k}^{\alpha} \lambda_{i}^{\alpha} K_{k i i k} \\
& =\frac{1}{2} \sum_{i, k}\left(\lambda_{i}-\lambda_{k}\right)^{2} K_{i k i k} \geq \frac{1}{2} a \sum_{i, k}\left(\lambda_{i}-\lambda_{k}\right)^{2}=n a \operatorname{tr} H_{\alpha}^{2} .
\end{aligned}
$$

Substituting (3.5) and (3.6) into (3.4), we obtain

$$
\begin{aligned}
B & \geq \sum_{\alpha}\left[n a \operatorname{tr} H_{\alpha}^{2}-\frac{1}{3}(n-1)^{1 / 2}(b-a) \sum_{\beta \neq \alpha} \operatorname{tr} H_{\beta}^{2}\right. \\
& \left.-\frac{1}{3}(n-1)^{1 / 2}(p-1)(b-a) \operatorname{tr} H_{\alpha}^{2}\right] \\
& =n b S-\left[n+\frac{2}{3}(p-1)(n-1)^{1 / 2}\right](b-a) S .
\end{aligned}
$$

This proves Lemma 2.

We shall next estimate the integral of $C$.

Lemma 3. $\int_{M} C \geq-\frac{1}{72} p n(n-1)(26 n-25) \int_{M}(b-a)^{2}$.

Proof. Note that

$$
\begin{aligned}
& -\sum_{i, j, k, \alpha}\left(h_{i k}^{\alpha} K_{\alpha j i j k}+h_{i j}^{\alpha} K_{\alpha i j k k}\right) \\
& \quad=-\sum_{i, j, k, \alpha} \nabla_{k}\left(h_{i k}^{\alpha} K_{\alpha j i j}+h_{i j}^{\alpha} K_{\alpha i j k}\right)+\sum_{i, j, k, \alpha}\left(h_{i k k}^{\alpha} K_{\alpha j i j}+h_{i j k}^{\alpha} K_{\alpha i j k}\right) .
\end{aligned}
$$

We define a differentiable 1 -form as

$$
\omega=\sum_{i, j, k, \alpha}\left(h_{i k}^{\alpha} K_{\alpha j i j}+h_{i j}^{\alpha} K_{\alpha i j k}\right) \omega_{k}
$$

It follows that

$$
\operatorname{div} \omega=\sum_{i, j, k, \alpha} \nabla_{k}\left(h_{i k}^{\alpha} K_{\alpha j i j}+h_{i j}^{\alpha} K_{\alpha i j k}\right)
$$

Thus

$$
C=\sum_{i, j, k, \alpha}\left(h_{i j k}^{\alpha}\right)^{2}+\sum_{i, j, k, \alpha}\left(h_{i k k}^{\alpha} K_{\alpha j i j}+h_{i j k}^{\alpha} K_{\alpha i j k}\right)-\operatorname{div} \omega
$$


Since $M$ is minimal, we have

$$
\sum_{i} h_{i i j}^{\alpha}=0 \text { for all } j, \alpha .
$$

From (2.5), (3.9), and Proposition 2, we have

$$
\begin{aligned}
\sum_{i, j, k, \alpha} h_{i k k}^{\alpha} K_{\alpha j i j} & =\sum_{i, j, k, \alpha}\left(h_{k k i}^{\alpha}-K_{\alpha k i k}\right) K_{\alpha j i j}=-\sum_{i, \alpha}\left(\sum_{j} K_{\alpha j i j}\right)^{2} \\
& \geq-\frac{1}{4} p n(n-1)^{2}(b-a)^{2} .
\end{aligned}
$$

On the other hand, by Proposition 2, we have

$$
\begin{aligned}
& \sum_{i, j, k, \alpha}\left(h_{i j k}^{\alpha}\right)^{2}+\sum_{i, j, k, \alpha} h_{i j k}^{\alpha} K_{\alpha i j k} \\
& \geq-\frac{1}{4} \sum_{i, j, k, \alpha}\left(K_{\alpha i j k}\right)^{2} \\
& \geq-\frac{1}{4} \sum_{\alpha} \sum_{i, j, k \text { distinct }}\left(K_{\alpha i j k}\right)^{2}-\frac{1}{2} \sum_{\alpha} \sum_{i \neq j}\left(K_{\alpha i j i}\right)^{2} \\
& \geq-\frac{1}{9} p n(n-1)(n-2)(b-a)^{2}-\frac{1}{8} p n(n-1)(b-a)^{2} .
\end{aligned}
$$

So

$$
C \geq-\frac{1}{72} p n(n-1)(26 n-25)(b-a)^{2}-\operatorname{div} \omega,
$$

and by using Green's divergence theorem, we get

$$
\int_{M} C \geq-\frac{1}{72} p n(n-1)(26 n-25) \int_{M}(b-a)^{2} \text {. }
$$

Lemma 3 follows.

Now we define

$$
\begin{aligned}
& D(n, p)=n+\frac{2}{3}(p-1)(n-1)^{1 / 2} \\
& E(n, p)=\frac{1}{72} p n(n-1)(26 n-25) .
\end{aligned}
$$

Theorem 1 (Generalized Simons inequality). Let $M^{n}$ be an $n$-dimensional oriented closed minimal submanifold in an $(n+p)$-dimensional Riemannian manifold $N^{n+p}$. Denote the infimum and the supremum of the sectional curvature of $N$ at a point $x$ by $a(x)$ and $b(x)$ respectively. Then

$$
\int_{M}\left[n b S-\left(1+\frac{1}{2} \operatorname{sgn}(p-1)\right) S^{2}-D(n, p)(b-a) S-E(n, p)(b-a)^{2}\right] \leq 0 .
$$

Proof. Combining Proposition 1, Lemma 1 and 2, we obtain

(3.14) $\frac{1}{2} \Delta S \geq n b S-\left(1+\frac{1}{2} \operatorname{sgn}(p-1)\right) S^{2}-\left[n+\frac{2}{3}(p-1)(n-1)^{1 / 2}\right](b-a) S+C$.

Integrating both sides of (3.14) and applying Lemma 3 , we have

$$
\int_{M}\left[n b S-\left(1+\frac{1}{2} \operatorname{sgn}(p-1)\right) S^{2}-D(n, p)(b-a) S-E(n, p)(b-a)^{2}\right] \leq 0
$$

This completes the proof of Theorem 1. 
Denote

$$
\begin{gathered}
\alpha(n, p)=\frac{1}{12}[p n(n-1)(52 n-50)]^{1 / 2}, \\
\beta(n, p)=n+\frac{2}{3}(p-1)(n-1)^{1 / 2}+\frac{1}{12}[p n(n-1)(52 n-50)]^{1 / 2} .
\end{gathered}
$$

We are now in a position to prove

Theorem 2. There is a number $\delta(n, p)$ with $0<\delta(n, p)<1$ such that if there exists an oriented closed minimal submanifold $M^{n}$ in a complete simply connected manifold $N^{n+p}$ with $\delta(n, p) \leq K_{N} \leq 1$ and

$$
\alpha(n, p)(1-c) \leq S \leq n-\frac{1}{3} n \operatorname{sgn}(p-1)-\beta(n, p)(1-c),
$$

where $c$ is the infimum of the sectional curvature of $N$, then either $M$ is the unit sphere $S^{n}(1)$, one of the Clifford minimal hypersurfaces $S^{k}(\sqrt{k / n}) \times$ $S^{n-k}(\sqrt{(n-k) / n}), k=1,2, \ldots, n-1$, in $S^{n+1}(1)$, or the Veronese surface in $S^{4}(1)$. Moreover, $N=S^{n+p}(1)$.

Proof. Since

$$
c \leq a(x) \leq b(x) \leq 1
$$

(3.15) gives

$$
\int_{M}\left[n S-\left(1+\frac{1}{2} \operatorname{sgn}(p-1)\right) S^{2}-D(n, p)(1-c) S-E(n, p)(1-c)^{2}\right] \leq 0
$$

Take

$$
\delta(n, p)=1-n(3-\operatorname{sgn}(p-1))\left(3 D(n, p)+6 E^{1 / 2}(n, p)\right)^{-1}
$$

Then

$$
\alpha(n, p)(1-c) \leq n-\frac{1}{3} n \operatorname{sgn}(p-1)-\beta(n, p)(1-c) .
$$

From the assumption

$$
\alpha(n, p)(1-c) \leq S \leq n-\frac{1}{3} n \operatorname{sgn}(p-1)-\beta(n, p)(1-c),
$$

we see that

$$
n S-\left(1+\frac{1}{2} \operatorname{sgn}(p-1)\right) S^{2}-D(n, p)(1-c) S-E(n, p)(1-c)^{2} \geq 0 .
$$

Therefore, all inequalities in (3.10), (3.11), (3.15), and (3.18) are actually equalities. This implies $1-c=b-a=0$ and $N$ is a complete simply connected Riemannian manifold with constant curvature 1 . Hence $N=S^{n+p}(1)$. This together with (3.16) and (3.18) gives

$$
S=0 \text { or } S=n-\frac{1}{3} n \operatorname{sgn}(p-1) .
$$

Furthermore, the previous inequalities become equalities, and it is not hard to see from Proposition 1 that either $M$ is the unit sphere $S^{n}(1)$, one of the Clifford hypersurfaces $S^{k}(\sqrt{k / n}) \times S^{n-k}(\sqrt{(n-k) / n}), k=1,2, \ldots, n-1$, or the Veronese surface. This proves Theorem 2.

Remark 1 . Theorem 2 can be considered as a generalization of the main theorems of $[2,6]$ as well as a pinching theorem for ambient manifolds. 
Theorem 3. Let $M^{n}$ be an oriented closed minimal submanifold with parallel second fundamental form in a Riemannian manifold $N^{n+p}$. Then

(i) $S \leq p n d+F(n, p)(d-c)$, where $F(n, p)=\frac{2}{3} p(p-1)(n-1)^{1 / 2}$ and $d$ is the supremum of the sectional curvature of $N$,

(ii) if $\delta^{\prime}(n, p)<K_{N} \leq 1$, here

$$
\delta^{\prime}(n, p)=1-n(3-\operatorname{sgn}(p-1))\left[3 n+2(p-1)(n-1)^{1 / 2}\right]^{-1},
$$

then either $M$ is totally geodesic or $n-\frac{1}{3} n \operatorname{sgn}(p-1)-D(n, p)(1-c) \leq S \leq$ $p n+F(n, p)(1-c)$.

Proof. From the proof of Lemma 3 we have

$$
C=-\sum_{i, j, k, \alpha} \nabla_{k}\left(h_{i k}^{\alpha} K_{\alpha j i j}+h_{i j}^{\alpha} K_{\alpha i j k}\right) .
$$

It is easy to see from (2.5) that $K_{\alpha i j k}=0$, for all $i, j, k, \alpha$. So

$$
C=0 \text {. }
$$

Since $\frac{1}{2} \Delta S=\sum\left(h_{i j k}^{\alpha}\right)^{2}+\sum h_{i j}^{\alpha} \Delta h_{i j}^{\alpha}=0, S$ is a constant. This together with (3.3) and (3.9) implies

$$
A+B=0 .
$$

Obviously,

$$
\sum_{\alpha, \beta} N\left(H_{\alpha} H_{\beta}-H_{\beta} H_{\alpha}\right)+\sum_{\alpha} S_{\alpha}^{2} \geq S^{2} / p .
$$

For fixed $\alpha$, similar to the estimate of lower bound for $B$, we have

$$
\begin{aligned}
\text { LHS of (3.4) }= & \sum_{i, k}\left(\lambda_{i}^{\alpha}\right)^{2} K_{i k i k}+\sum_{i, k} \lambda_{k}^{\alpha} \lambda_{i}^{\alpha} K_{k i i k}+\sum_{i, k, \beta} h_{k i}^{\beta} \lambda_{i}^{\alpha} K_{\alpha \beta k i} \\
\leq & n d \operatorname{tr} H_{\alpha}^{2}+\frac{1}{3}(p-1)(n-1)^{1 / 2}(d-c) \operatorname{tr} H_{\alpha}^{2} \\
& +\frac{1}{3}(n-1)^{1 / 2}(d-c) \sum_{\beta \neq \alpha} \operatorname{tr} H_{\beta}^{2} .
\end{aligned}
$$

This gives

$$
B \leq n d S+\frac{2}{3}(p-1)(n-1)^{1 / 2}(d-c) S .
$$

It follows from (3.20), (3.21), and (3.22) that

$$
n d S+\frac{2}{3}(p-1)(n-1)^{1 / 2}(d-c) S \geq S^{2} / p .
$$

This yields

$$
S \leq p n d+\frac{2}{3} p(p-1)(n-1)^{1 / 2}(d-c) .
$$

If $\delta^{\prime}(n, p)<K_{N} \leq 1$, it is not hard to see from the definition of $\delta^{\prime}(n, p)$ that

$$
n-\frac{1}{3} n \operatorname{sgn}(p-1)-D(n, p)(1-c)>0 .
$$

By (3.20), Proposition 1, and Lemma 2, we get

$$
n S-\left(1+\frac{1}{S} \operatorname{sgn}(p-1)\right) S^{2}-D(n, p)(1-c) S \leq 0,
$$


which together with (3.23) implies that either $S=0$ or $n-\frac{1}{3} n \operatorname{sgn}(p-1)-$ $D(n, p)(1-c) \leq S \leq p n+F(n, p)(1-c)$. This completes the proof of Theorem 3.

Remark 2. When $p=1$, the constant $\delta^{\prime}(n, p)$ equals zero, which is independent of dimension. In this case, we have that either $S=0$ or $n c \leq S \leq n$.

\section{ACKNOWLEDGMENT}

The author would like to express his gratitude to Professor Hesheng $\mathrm{Hu}$ and Chaohao $\mathrm{Gu}$ for their constant advice and helpful discussions. Thanks also to the referee for valuable suggestions.

\section{REFERENCES}

1. Q. M. Cheng and H. C. Yang, A note on the pinching constant of minimal hypersurfaces with constant scalar curvature in unit sphere, Chinese Sci. Bull. 36 (1991), 1-6.

2. S. S. Chern, M. do Carmo, and S. Kobayashi, Minimal submanifolds of a sphere with second fundamental form of constant length, Functional Analysis and Related Fields (F. E. Browder, ed.), Springer, New York, 1970.

3. H. Gauchman, Minimal submanifolds of a sphere with bounded second fundamental form, Trans. Amer. Math. Soc. 298 (1986), 777-791.

4. S. I. Goldberg, Curvature and homology, Academic Press, London, 1962.

5. H. B. Lawson, Rigidity theorems in rank-1 symmetric space, J. Differential Geom. 4 (1970), 349-357.

6. A. M. Li and J. M. Li, An intrinsic rigidity theorem for minimal submanifolds in a sphere, Arch. Math. 58 (1992), 582-594.

7. M. Okumura, Hypersurface and a pinching problem on the second fundamental tensor, Amer. J. Math. 96 (1974), 207-213.

8. C. K. Peng and C. L. Terng, Minimal hypersurfaces of sphere with constant scalar curvature, Sem. of Minimal Submanifolds (E. Bombieri, ed.), Princeton Univ. Press, Princeton, N.J., 1983.

9. Y. B. Shen, On intrinsic rigidity for minimal submanifolds in a sphere, Sci. Sinica Ser. A 32 (1989), 769-781.

10. J. Simons, Minimal varieties in Riemannian manifolds, Ann. of Math. (2) 88 (1968), 62-105.

11. H. W. Xu, Some results on geometry of Riemannian submanifolds, Ph.D. dissertation, Fudan Univ., 1990.

12. S. T. Yau, Submanifolds with constant mean curvature. I, II, Amer. J. Math. 96 (1974), 346-366; 97 (1975), 76-100.

Center for Mathematical Sciences, Zhejiang University, Hangzhou 310027, People's REPUBLIC OF ChINA

Current address: Department of Mathematics, Faculty of Science, Kyushu University, Fukuoka 812, Japan 